CRYSTALLOGRAPHIC COMMUNICATIONS

ISSN 2056-9890

Received 7 May 2019

Accepted 28 May 2019

Edited by C. Massera, Università di Parma, Italy

Keywords: crystal structure; N,N,N-trimethyl-1(4-vinylphenyl)methanaminium cation; 4-vinylbenzenesulfonate anion; hydrogen bonds; Hirshfeld surface analysis.

CCDC reference: 1919325

Supporting information: this article has supporting information at journals.iucr.org/e

\section{Structure and Hirshfeld surface analysis of the salt $\mathrm{N}, \mathrm{N}, \mathrm{N}$-trimethyl-1-(4-vinylphenyl)methanaminium 4-vinylbenzenesulfonate}

\author{
C. John McAdam, Lyall R. Hanton, Stephen C. Moratti, Jim Simpson* and \\ Ravindra N. Wickramasinhage
}

Department of Chemistry, University of Otago, PO Box 56, Dunedin, New Zealand. *Correspondence e-mail: jsimpson@alkali.otago.ac.nz

In the title compound, the asymmetric unit comprises an $N, N, N$-trimethyl-1-(4vinylphenyl)methanaminium cation and a 4-vinylbenzenesulfonate anion, $\mathrm{C}_{12} \mathrm{H}_{18} \mathrm{~N}^{+} \cdot \mathrm{C}_{8} \mathrm{H}_{7} \mathrm{O}_{3} \mathrm{~S}^{-}$. The salt has a polymerizable vinyl group attached to both the cation and the anion. The methanaminium and vinyl substituents on the benzene ring of the cation subtend angles of 86.6 (3) and $10.5(9)^{\circ}$ to the ring plane, while the anion is planar excluding the sulfonate $\mathrm{O}$ atoms. The vinyl substituent on the benzene ring of the cation is disordered over two sites with a refined occupancy ratio of $0.542(11): 0.458(11)$. In the crystal, C-H $\cdots \mathrm{O}$ hydrogen bonds dominate the packing and combine with a $\mathrm{C}-\mathrm{H} \cdots \pi$ (ring) contact to stack the cations and anions along the $a$-axis direction. Hirshfeld surface analysis of the salt and of the individual cation and anion components is also reported.

\section{Chemical context}

Hydrogels continue to be the subject of intense study, particularly with regard to biomedical applications and new technologies (Van Vlierberghe et al., 2011; Sun et al., 2015; Goswami et al., 2017; Pushparajan et al., 2018). Limiting development has been the poor mechanical strength of conventional hydrogel formulations. Numerous strategies, singly and in combination, have been utilized in efforts to improve toughness and stretchability, and the results have been extensively reviewed (Naficy et al., 2011; Peak et al., 2013; Zhao, 2014). Our current approach is to build in capacity for self-healing, and exploits polyampholytes (Zurick \& Bernards, 2014), polymers formed from the covalent cross-linking of mixed cationic and anionic monomers. The title compound is one such set of ion-pair co-monomers, simply prepared from commercially available trimethylammonium cation and sulfonate anion salts.

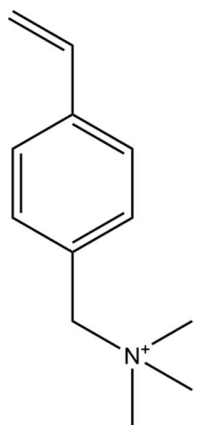

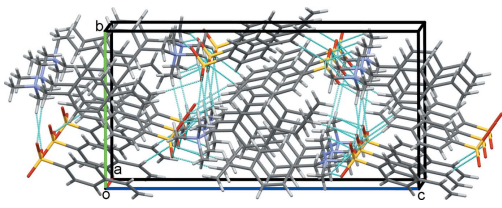


Table 1

Hydrogen-bond geometry $\left(\AA,^{\circ}\right)$.

$\mathrm{Cg} 1$ is the centroid of the $\mathrm{C} 1-\mathrm{C} 6$ benzene ring.

\begin{tabular}{lllll}
\hline$D-\mathrm{H} \cdots A$ & $D-\mathrm{H}$ & $\mathrm{H} \cdots A$ & $D \cdots A$ & $D-\mathrm{H} \cdots A$ \\
\hline $\mathrm{C} 14-\mathrm{H} 14 B \cdots \mathrm{O} 3$ & 0.98 & 2.32 & $3.264(5)$ & 161 \\
$\mathrm{C} 14-\mathrm{H} 14 A \cdots \mathrm{O} 2^{\mathrm{i}}$ & 0.98 & 2.48 & $3.346(5)$ & 147 \\
$\mathrm{C} 15-\mathrm{H} 15 A \cdots \mathrm{O} 1^{\mathrm{i}}$ & 0.98 & 2.63 & $3.544(4)$ & 155 \\
$\mathrm{C} 15-\mathrm{H} 15 A \cdots \mathrm{O} 2^{\mathrm{i}}$ & 0.98 & 2.49 & $3.348(4)$ & 147 \\
$\mathrm{C} 13-\mathrm{H} 13 B \cdots 3^{\mathrm{ii}}$ & 0.99 & 2.56 & $3.466(5)$ & 152 \\
$\mathrm{C} 15-\mathrm{H} 15 B \cdots \mathrm{O} 2^{\mathrm{ii}}$ & 0.98 & 2.60 & $3.477(4)$ & 149 \\
$\mathrm{C} 16-\mathrm{H} 16 B \cdots \mathrm{O} 1^{\text {iii }}$ & 0.98 & 2.61 & $3.365(4)$ & 134 \\
$\mathrm{C} 16-\mathrm{H} 16 C \cdots \mathrm{O} 2^{\mathrm{i}}$ & 0.98 & 2.52 & $3.370(5)$ & 146 \\
$\mathrm{C} 41-\mathrm{H} 41 \cdots \mathrm{O}^{2}{ }^{\mathrm{i}}$ & 0.95 & 2.58 & $3.481(4)$ & 157 \\
$\mathrm{C} 42-\mathrm{H} 42 B \cdots \mathrm{O} 1^{\mathrm{v}}$ & 0.95 & 2.63 & $3.494(4)$ & 151 \\
$\mathrm{C} 5-\mathrm{H} 5 \cdots C g 1^{\text {iv }}$ & 0.95 & 2.93 & $3.837(4)$ & 161 \\
\hline
\end{tabular}

Symmetry codes: (i) $x-1, y, z$; (ii) $-x+1, y+\frac{1}{2},-z+\frac{1}{2}$; (iii) $-x+1, y-\frac{1}{2},-z+\frac{1}{2}$; (iv) $x-\frac{1}{2},-y+\frac{1}{2},-z ;(\mathrm{v})-x+\frac{3}{2},-y+1, z-\frac{1}{2}$.

\section{Structural commentary}

The asymmetric unit of the title salt, (I), comprises an $N, N, N$ trimethyl-1-(4-vinylphenyl)methanaminium cation and a 4-vinylbenzenesulfonate anion, linked by a C14-H14B . OO3 hydrogen bond (Table 1) between a methyl group of the trimethylmethanaminium unit and a sulfonate oxygen, Fig. 1. The vinyl substituent on the benzene ring of the cation is disordered over two sites with a refined occupancy ratio of 0.542 (11):0.458 (11). In the cation, the C7/C13/N1 and C10/ $\mathrm{C} 101 / \mathrm{C} 102$ planes of the methanaminium and major vinyl substituents on the benzene ring subtend angles of 86.6 (3) and $10.5(9)^{\circ}$, respectively, to the ring plane. In contrast, excluding the sulfonate $\mathrm{O}$ atoms, the $\mathrm{S}$ and ordered vinyl substituents lie close to the benzene ring plane in the anion with an r.m.s. deviation of $0.0753 \AA$ from the S1/C1-C6/C41/ C42 plane.

\section{Supramolecular features}

Packing in this salt is dominated by an extensive number of $\mathrm{C}-\mathrm{H} \cdots \mathrm{O}$ hydrogen bonds, Table $1 . \mathrm{O} 2$ acts as a trifurcated acceptor forming $\mathrm{C} 14-\mathrm{H} 14 A \cdots \mathrm{O} 2^{\mathrm{i}}, \mathrm{C} 15-\mathrm{H} 15 A \cdots \mathrm{O} 2^{\mathrm{i}}$ and $\mathrm{C} 16-\mathrm{H} 16 C \cdots \mathrm{O} 2^{\mathrm{i}}$ hydrogen bonds [symmetry code: (i) $x-1$, $y, z]$. $\mathrm{C} 14$ and $\mathrm{C} 15$ are bifurcated donors with the $\mathrm{C} 15-$ $\mathrm{H} 15 A \cdots \mathrm{O} 1^{\mathrm{i}}$ and $\mathrm{C} 15-\mathrm{H} 15 A \cdots \mathrm{O} 2^{\mathrm{i}}$ contacts forming $R_{1}^{2}(4)$

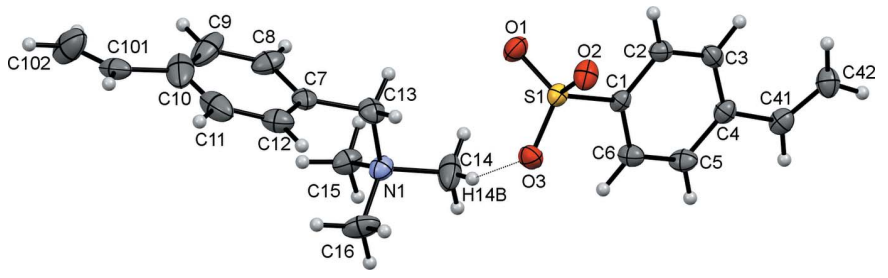

Figure 1

The asymmetric unit of the title compound showing the atom numbering with ellipsoids drawn at the $50 \%$ probability level. The $\mathrm{C}-\mathrm{H} \cdots \mathrm{O}$ hydrogen bond linking the two components is drawn as a dotted black line. For clarity, only the major disorder component of the vinyl substituent on the benzene ring of the cation is shown.

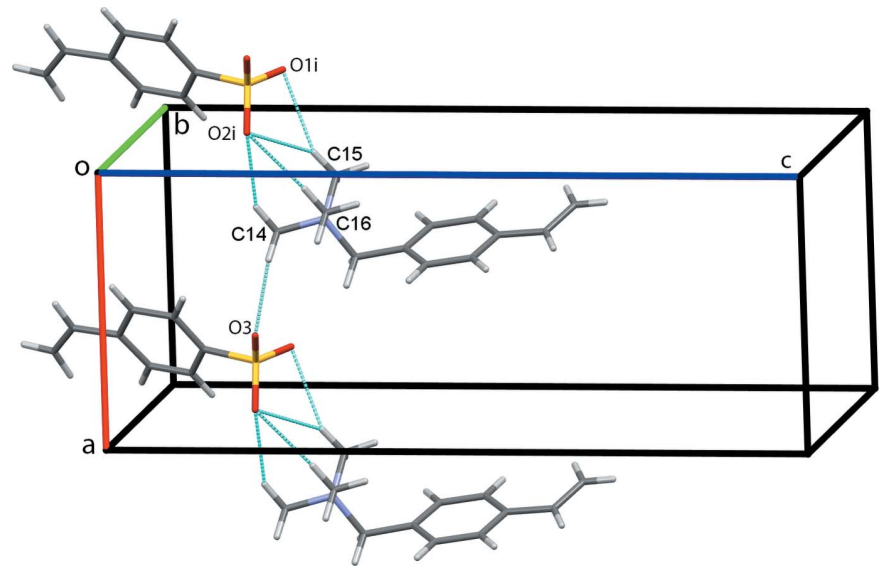

Figure 2

Chains of cations and anions of (I) along the $a$ axis. Hydrogen bonds are shown as cyan dotted lines [symmetry code: (i) $x-1, y, z$ ].

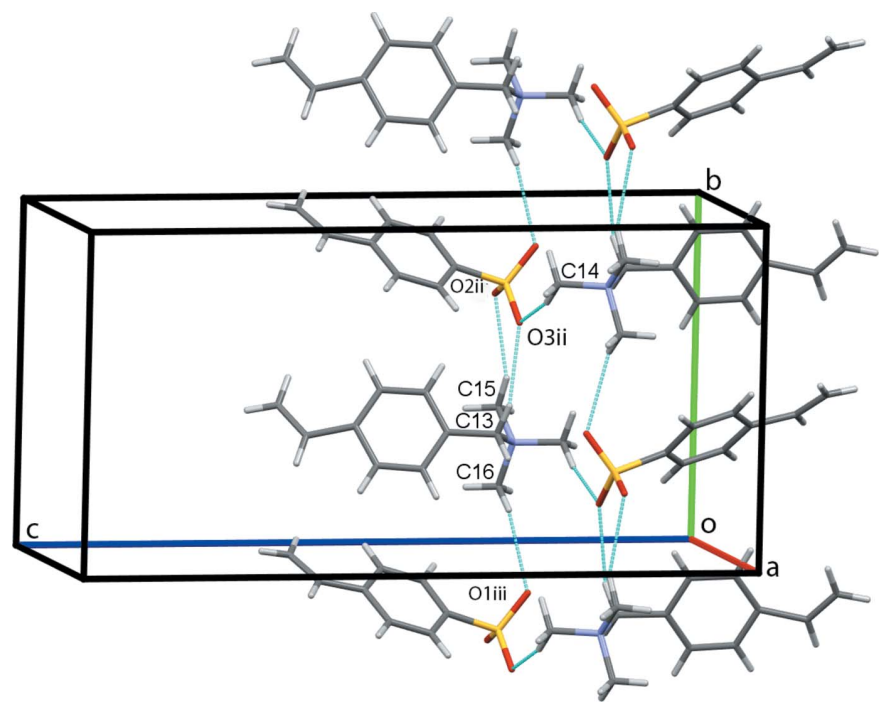

Figure 3

Double chains of cation-anion dimers along $b$. Hydrogen bonds are shown as cyan dotted lines [symmetry codes: (ii) $1-x, \frac{1}{2}+y, \frac{1}{2}-z$; (iii) $\left.1-x,-\frac{1}{2}+y, \frac{1}{2}-z\right]$.

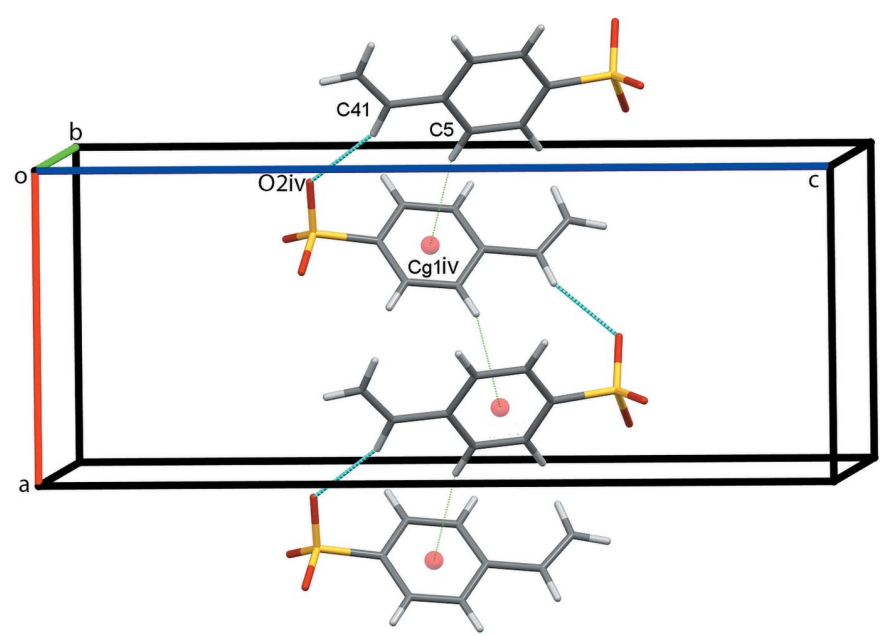

Figure 4

Chains of anions along $a$. Hydrogen bonds and $\mathrm{C}-\mathrm{H} \cdots \pi$ interactions are shown as cyan and green dotted lines, respectively [symmetry code: (iv) $\left.x-\frac{1}{2}, \frac{1}{2}-y,-z\right]$. 


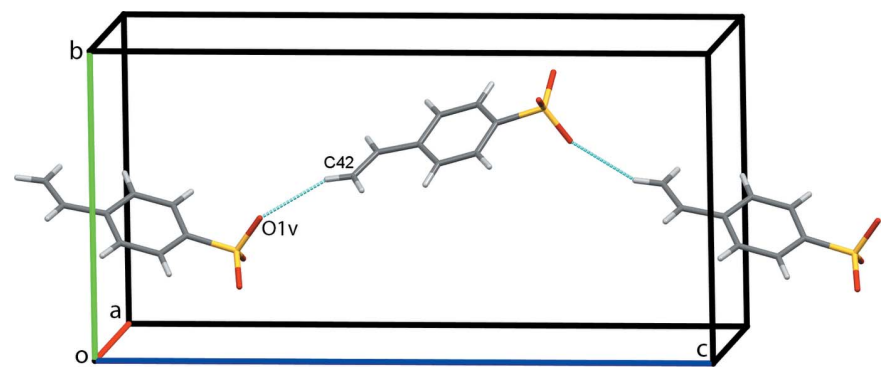

Figure 5

Zigzag chains of anions along $c$. Hydrogen bonds are shown as cyan dotted lines [symmetry code: (v) $\frac{3}{2}-x, 1-y, z-\frac{1}{2}$ ].

ring motifs. $\mathrm{C} 14-\mathrm{H} 14 B \cdots \mathrm{O} 3$ contacts link the cation-anion pairs into chains along the $a$-axis direction, Fig. 2. Cationanion dimers are generated by $\mathrm{C} 13-\mathrm{H} 13 B \cdots \mathrm{O}{ }^{\mathrm{ii}}$ and $\mathrm{C} 15-$ $\mathrm{H} 15 B \cdots \mathrm{O} 2^{\mathrm{ii}}$ contacts with adjacent dimers linked into columns along $b$ by $\mathrm{C} 16-\mathrm{H} 16 B \cdots \mathrm{O} 1^{\mathrm{iii}}$ hydrogen bonds [symmetry codes: (ii) $1-x, \frac{1}{2}+y, \frac{1}{2}-z$; (iii) $1-x,-\frac{1}{2}+y, \frac{1}{2}-z$ ]. Additional $\mathrm{C} 14-\mathrm{H} 14 B$. . O3 hydrogen bonds form double columns along $b$ with the vinyl substituents of the proximate cations and anions pointing in opposite directions, Fig. 3. Chains of anions form along $a$ through $\mathrm{C} 41-\mathrm{H} 41 \cdots \mathrm{O} 2^{\text {iv }}$ hydrogen bonds augmented by $\mathrm{C} 5-\mathrm{H} 5 \cdots \mathrm{Cg} 1^{\text {iv }}$ contacts, Fig. 4 [symmetry code: (iv) $x-\frac{1}{2}, \frac{1}{2}-y,-z$ ]. Finally, weak C42$\mathrm{H} 42 \mathrm{~B} \cdots \mathrm{O} 1^{\mathrm{v}}$ hydrogen bonds link the anions in a head-to-tail fashion into zigzag chains along $c$, Fig. 5 [symmetry code: (v) $\left.\frac{3}{2}-x, 1-y, z-\frac{1}{2}\right]$. This extensive series of contact combines to assemble an extended network structure with the cations and anions stacked along the $a$-axis direction, Fig. 6 .

\section{Hirshfeld surface analysis}

Further details of the intermolecular architecture of this salt were obtained using Hirshfeld surface analysis (Spackman \& Jayatilaka, 2009) with surfaces and two-dimensional fingerprint plots generated by CrystalExplorer (Turner et al., 2017).

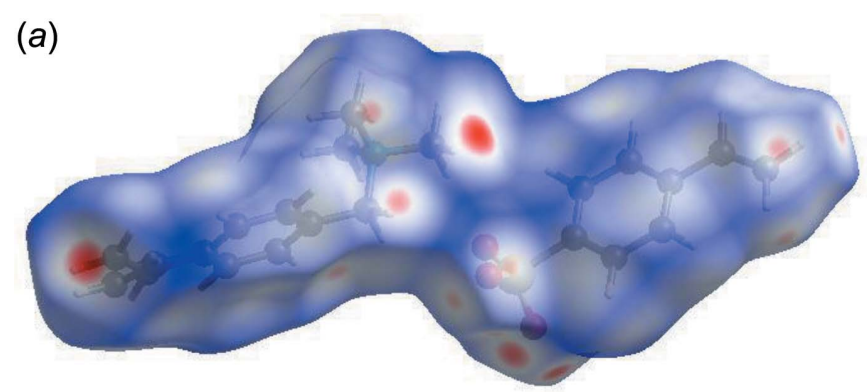

(b)

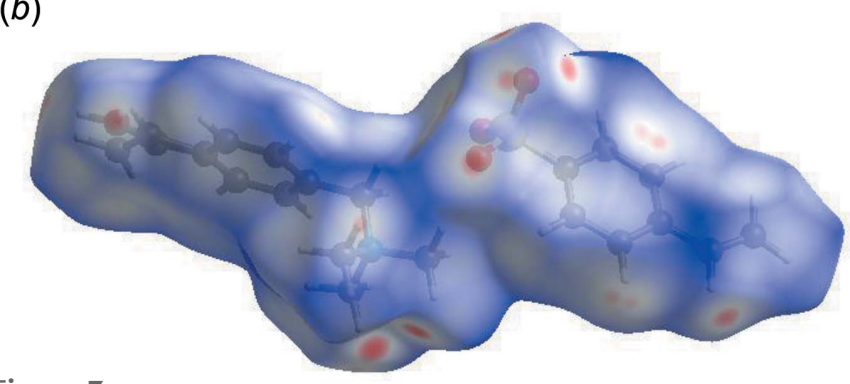

Figure 7

Hirshfeld surfaces of (1) viewed for opposite faces of the salt.

Hirshfeld surfaces viewed for opposite faces of the complete salt are shown in Fig. 7. Both disorder components are included in these surface calculations. The red circles on the Hirshfeld surfaces correspond to the numerous $\mathrm{C}-\mathrm{H} \cdots \mathrm{O}$ contacts that play a significant role in stabilizing the packing in this structure. Fingerprint plots of the principal contacts on the Hirshfeld surface of the salt are shown in Fig. 8. These comprise $\mathrm{H} \cdots \mathrm{H}, \mathrm{H} \cdots \mathrm{C} / \mathrm{C} \cdots \mathrm{H}$, and $\mathrm{H} \cdots \mathrm{O} / \mathrm{O} \cdots \mathrm{H}$ contacts. The much less significant $\mathrm{C} \cdots \mathrm{C}$ and $\mathrm{H} \cdots \mathrm{S} / \mathrm{S} \cdots \mathrm{H}$ contributions are not shown in the figure but are detailed in Table 2.

It is also instructive to investigate the differences in contacts for the discrete cation and anion components of (I) by recording fingerprint plots of the cation and anion individually. All of the surface contributions for the cation and anion are also shown in Table 2, with fingerprint plots for principal contacts found in the individual cation and anion also

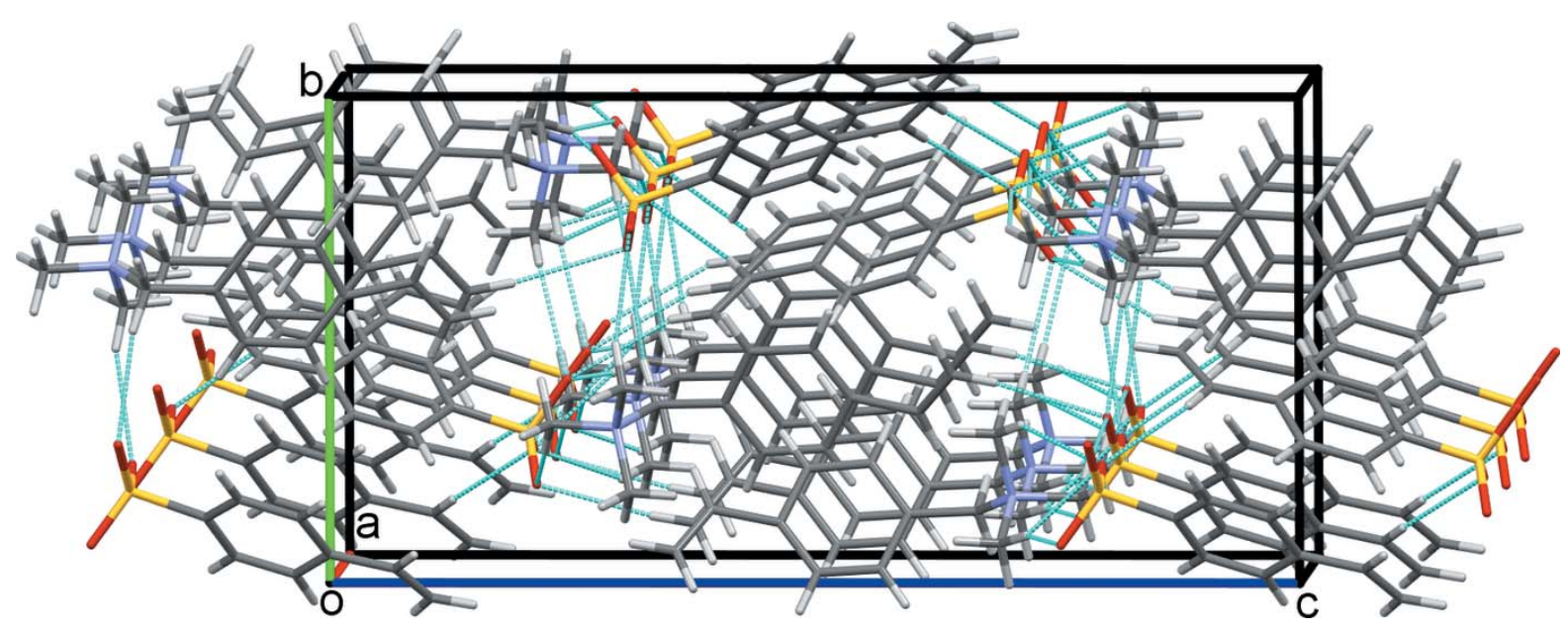

Figure 6

Overall packing for (I) viewed along the $a$-axis direction. 
Table 2

Percentage contributions of interatomic contacts to the Hirshfeld surface for (I).

\begin{tabular}{llll}
\hline Contacts & \multicolumn{2}{l}{ Included surface area } \\
\hline & Salt & Cation & Anion \\
$\mathrm{H} \cdots \mathrm{H}$ & 52.5 & 60.3 & 37.9 \\
$\mathrm{H} \cdots \mathrm{C} / \mathrm{C} \cdots \mathrm{H}$ & 26.1 & 20.8 & 27.8 \\
$\mathrm{H} \cdots \mathrm{O} / \mathrm{O} \cdots \mathrm{H}$ & 20.7 & 17.8 & 34.2 \\
$\mathrm{C} \cdots \mathrm{C}$ & 0.5 & 0.9 & 0.0 \\
$\mathrm{H} \cdots \mathrm{S} / \mathrm{S} \cdots \mathrm{H}$ & 0.1 & 0.1 & 0.1 \\
\hline
\end{tabular}

displayed in Fig. 8. The most notable differences between the values for the salt and its components are that the $\mathrm{H} \cdots \mathrm{H}$ van der Waals interactions increase significantly for the cation, while the anion shows considerable increases in the $\mathrm{H} \cdots \mathrm{O}$ / $\mathrm{O} \cdots \mathrm{H}$ and $\mathrm{H} \cdots \mathrm{C} / \mathrm{C} \cdots \mathrm{H}$ contacts. These differences reflect the fact that, whereas the contacts for the cations are limited to cation-anion interactions, the anions are also involved in distinct anion-anion contacts, vide supra. The C...C and

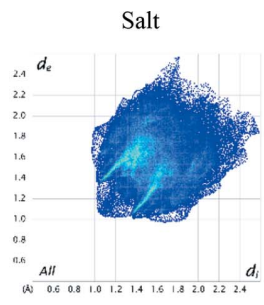

(a)

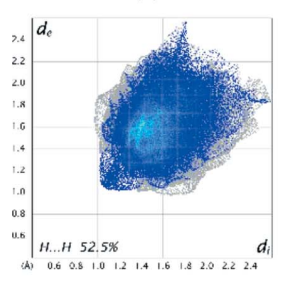

(d)

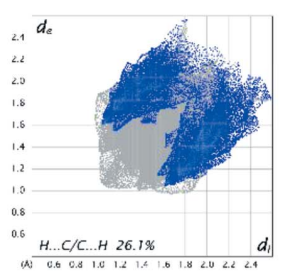

(g)

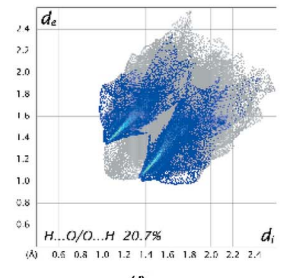

(j)

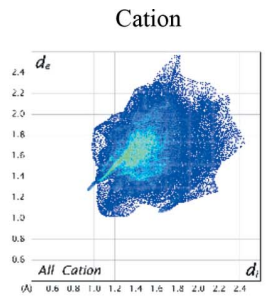

(b)

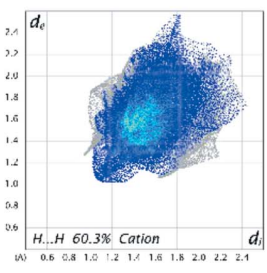

(e)

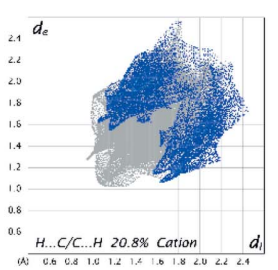

(h)

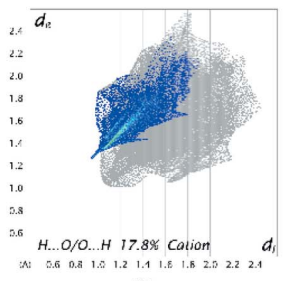

(k)

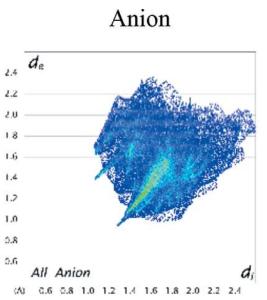

(c)

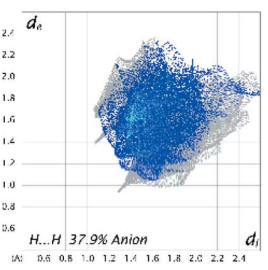

(f)

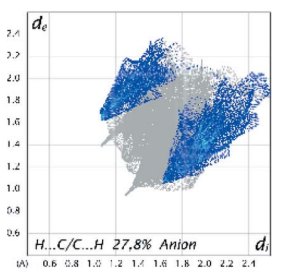

(i)

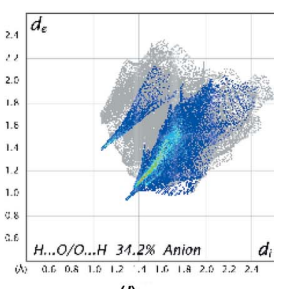

(I)
Figure 8

Full two-dimensional fingerprint plots for the salt $(a)$, cation $(b)$ and anion $(c)$ together with $(d)-(l)$ separate principal contact types for the salt, cation and anion systems respectively. These are found to be $\mathrm{H} \cdots \mathrm{H}$, $\mathrm{H} \cdots \mathrm{C} / \mathrm{C} \cdots \mathrm{H}$, and $\mathrm{H} \cdots \mathrm{O} / \mathrm{O} \cdots \mathrm{H}$ contacts.
$\mathrm{H} \cdots \mathrm{S} / \mathrm{S} \cdots \mathrm{H}$ contributions to all of the surfaces are very weak but are included in Table 2 for completeness.

\section{Database survey}

A search of the Cambridge Structural Database (Version 5.40 November 2018 with one update; Groom et al., 2016) reveals the fact that the salt reported here is quite unusual. Only two structures involving the $N, N, N$-trimethyl(4-vinylphenyl)methylammonium cation acting as counter-ions to polymolybdate (QAJXEH) and poly-tungstate (QAJXAD) anions were found (Vorotnikov et al., 2015). Structures of salts of the 4-vinylbenzenesulfonate anion are slightly more abundant, with organic methylquinolinium (RUMGAJ; Lee et al., 2015) and 4-\{2-[4-(dimethylamino)phenyl]vinyl\}-1-methylpyridinium (SAPDAR; Vijay et al., 2012) cations and hexaaqua manganese, cobalt and nickel complex cations (SUVBOA, SUVBUG and SUVCAN; Leonard et al., 1999).

\section{Synthesis and crystallization}

The title compound was prepared via an argentometric mixing approach (Li et al., 2010) from the silver salt of 4-vinylbenzenesulfonic acid, Ag-VBS (Woeste et al., 1993; Sikkema et al., 2007) and (vinylbenzyl)trimethylammonium chloride, VBT-Cl (Sigma Aldrich). A suspension of Ag-VBS in water and equimolar amount of VBT-Cl were stirred 30 minutes. After filtration of the $\mathrm{AgCl}$ precipitate, the solution was freeze-dried and the ion-pair co-monomers recrystallized from chloroform as irregular colourless blocks.

ESI $\mathrm{MS}+\mathrm{ve}(\mathrm{m} / \mathrm{z}): 176.14 \quad\left[\mathrm{C}_{12} \mathrm{H}_{18} \mathrm{~N}\right]^{+} ;$-ve: 183.01 $\left[\mathrm{C}_{8} \mathrm{H}_{7} \mathrm{SO}_{3}\right]^{-} .{ }^{1} \mathrm{H}$ NMR $\left(400 \mathrm{MHz}, \mathrm{DMSO}-d_{6}\right): 5.95(d d, J=18$, $\left.1 \mathrm{~Hz}, 1 \mathrm{H}, \mathrm{VBT}=\mathrm{CH}_{2}\right), 5.38(d d, J=11,1 \mathrm{~Hz}, 1 \mathrm{H}, \mathrm{VBT}$ $\left.=\mathrm{CH}_{2}\right), 6.80(d d, J=18,11 \mathrm{~Hz}, 1 \mathrm{H}, \mathrm{VBT}-\mathrm{CH}=), 7.61 \& 7.50$ $[2 \times(d, J=8 \mathrm{~Hz}, 2 \mathrm{H}, \mathrm{VBT}$ benzene $\mathrm{H})], 4.51(s, 2 \mathrm{H}, \mathrm{VBT}$ $\left.\mathrm{CH}_{2}\right), 4.51\left(s, 2 \mathrm{H}, \mathrm{VBT} \mathrm{CH} \mathrm{CH}_{2}\right), 3.02\left(s, 9 \mathrm{H}, \mathrm{VBT} \mathrm{CH}_{3}\right) .5 .84(d d$, $\left.J=18,1 \mathrm{~Hz}, 1 \mathrm{H}, \mathrm{VBS}=\mathrm{CH}_{2}\right), 5.27(d d, J=11,1 \mathrm{~Hz}, 1 \mathrm{H}, \mathrm{VBS}$ $\left.=\mathrm{CH}_{2}\right), 6.73(d d, J=18,11 \mathrm{~Hz}, 1 \mathrm{H}, \mathrm{VBS}-\mathrm{CH}=), 7.57 \& 7.42$ $[2 \times(d, J=8 \mathrm{~Hz}, 2 \mathrm{H}, \mathrm{VBS}$ benzene $\mathrm{H})]$

\section{Refinement}

Crystal data, data collection and structure refinement details are summarized in Table 3. All $\mathrm{H}$ atoms were refined using a riding model with $d(\mathrm{C}-\mathrm{H})=0.95 \AA$ and $U_{\text {iso }}(\mathrm{H})=1.2 U_{\text {eq }}(\mathrm{C})$ for aromatic and vinyl $\mathrm{H}$ atoms, $d(\mathrm{C}-\mathrm{H})=0.99 \AA$ and $U_{\text {iso }}(\mathrm{H})$ $=1.2 U_{\text {eq }}(\mathrm{C})$ for methylene and $d(\mathrm{C}-\mathrm{H})=0.98 \AA$ and $U_{\text {iso }}(\mathrm{H})$ $=1.5 U_{\text {eq }}(\mathrm{C})$ for methyl $\mathrm{H}$ atoms. The vinyl substituent on the benzene ring of the cation is disordered over two sites $(\mathrm{C} 101=\mathrm{C} 102$ and $\mathrm{C} 103=\mathrm{C} 104)$ with a refined occupancy ratio of 0.542 (11):0.458 (11).

\section{Funding information}

We thank the NZ Ministry of Business, Innovation and Employment Science Investment Fund (grant No. UOO$\mathrm{X} 1206$ ) for support of this work and the University of Otago 
Table 3

Experimental details.

\begin{tabular}{ll}
\hline Crystal data & \\
Chemical formula & $\mathrm{C}_{12} \mathrm{H}_{18} \mathrm{~N}^{+} \cdot \mathrm{C}_{8} \mathrm{H}_{7} \mathrm{O}_{3} \mathrm{~S}^{-}$ \\
$M_{\mathrm{r}}$ & 359.47 \\
Crystal system, space group & Orthorhombic, $P 2_{1} 2_{1} 2_{1}$ \\
Temperature $(\mathrm{K})$ & 100 \\
$a, b, c(\AA)$ & $8.3344(3), 10.5937(4), 21.1228(8)$ \\
$V\left(\AA^{3}\right)$ & $1864.98(12)$ \\
$Z$ & 4 \\
Radiation type & $\mathrm{Cu} \mathrm{K \alpha}$ \\
$\mu\left(\mathrm{mm}^{-1}\right)$ & 1.69 \\
Crystal size $(\mathrm{mm})$ & $0.20 \times 0.18 \times 0.08$ \\
& \\
Data collection & Rigaku Oxford Diffraction Super- \\
Diffractometer & Nova, Dual, Cu at home/near, \\
& Atlas \\
& Multi-scan $(C r y s A l i s P R O ;$ Rigaku \\
Absorption correction & OD, 2018) \\
& $0.911,1.000$ \\
$T_{\text {min }}, T_{\text {max }}$ & $4767,3103,2784$ \\
No. of measured, independent and & \\
$\quad$ observed $[I>2 \sigma(I)]$ reflections & 0.029 \\
$R_{\text {int }}$ & 0.620 \\
(sin $\theta / \lambda)_{\text {max }}\left(\AA^{-1}\right)$ & \\
& \\
Refinement & $0.040,0.103,1.04$ \\
$R\left[F^{2}>2 \sigma\left(F^{2}\right)\right], w R\left(F^{2}\right), S$ & 3103 \\
No. of reflections & 248 \\
No. of parameters & 10 \\
No. of restraints & H-atom parameters constrained \\
H-atom treatment & $0.37,-0.29$ \\
$\Delta \rho_{\text {max }}, \Delta \rho_{\min }\left(\mathrm{e} \AA^{-3}\right)$ & Flack $x$ determined using 870 \\
Absolute structure & quotients $\left[\left(I^{+}\right)-\left(I^{-}\right)\right] /\left[\left(I^{+}\right)+\left(I^{-}\right)\right]$ \\
& $-0.040(19)$ \\
Absolute structure parameter & \\
\hline &
\end{tabular}

Computer programs: CrysAlis PRO (Rigaku OD, 2018), SHELXT (Sheldrick, 2015a), SHELXL2018 (Sheldrick, 2015b), TITAN (Hunter \& Simpson, 1999), Mercury (Macrae et al., 2008), enCIFer (Allen et al., 2004), PLATON (Spek, 2009), publCIF (Westrip, 2010) and $\operatorname{Win} G X$ (Farrugia, 2012).

for the purchase of the diffractometer. JS also thanks the Department of Chemistry, University of Otago for support of his work.

\section{References}

Allen, F. H., Johnson, O., Shields, G. P., Smith, B. R. \& Towler, M. (2004). J. Appl. Cryst. 37, 335-338.

Farrugia, L. J. (2012). J. Appl. Cryst. 45, 849-854.
Goswami, S. K., McAdam, C. J., Hanton, L. R. \& Moratti, S. C. (2017). Macromol. Rapid Commun. 38, 1700103.

Groom, C. R., Bruno, I. J., Lightfoot, M. P. \& Ward, S. C. (2016). Acta Cryst. B72, 171-179.

Hunter, K. A. \& Simpson, J. (1999). TITAN2000. University of Otago, New Zealand.

Lee, S.-H., Yoo, B.-W., Yun, H., Jazbinsek, M. \& Kwon, O.-P. (2015). J. Mol. Struct. 1100, 359-365.

Leonard, M. A., Squattrito, P. J. \& Dubey, S. N. (1999). Acta Cryst. C55, 35-39.

Li, G., Xue, H., Gao, C., Zhang, F. \& Jiang, S. (2010). Macromolecules, 43, 14-16.

Macrae, C. F., Bruno, I. J., Chisholm, J. A., Edgington, P. R., McCabe, P., Pidcock, E., Rodriguez-Monge, L., Taylor, R., van de Streek, J. \& Wood, P. A. (2008). J. Appl. Cryst. 41, 466-470.

Naficy, S., Brown, H. R., Razal, J. M., Spinks, G. M. \& Whitten, P. G. (2011). Aust. J. Chem. 64, 1007-1025.

Parsons, S., Flack, H. D. \& Wagner, T. (2013). Acta Cryst. B69, 249259.

Peak, C. W., Wilker, J. J. \& Schmidt, G. (2013). Colloid Polym. Sci. 291, 2031-2047.

Pushparajan, C., Goswami, S. K., McAdam, C. J., Hanton, L. R., Dearden, P. K., Moratti, S. C. \& Cridge, A. G. (2018). Electrophoresis, 39, 824-832.

Rigaku OD (2018). CrysAlis PRO. Rigaku Oxford Diffraction Ltd, Yarnton, England.

Sheldrick, G. M. (2015a). Acta Cryst. A71, 3-8.

Sheldrick, G. M. (2015b). Acta Cryst. C71, 3-8.

Sikkema, F. D., Comellas-Aragonès, M., Fokkink, R. G., Verduin, B. J. M., Cornelissen, J. J. L. M. \& Nolte, R. J. M. (2007). Org. Biomol. Chem. 5, 54-57.

Spackman, M. A. \& Jayatilaka, D. (2009). CrystEngComm, 11, 19-32. Spek, A. L. (2009). Acta Cryst. D65, 148-155.

Sun, Z., Lv, F., Cao, L., Liu, L., Zhang, Y. \& Lu, Z. (2015). Angew. Chem. Int. Ed. 54, 7944-7948.

Turner, M. J., McKinnon, J. J., Wolff, S. K., Grimwood, D. J., Spackman, P. R., Jayatilaka, D. \& Spackman, M. A. (2017). CrystalExplorer17. University of Western Australia, Nedlands, Western Australia; http://hirshfeldsurface.net.

Van Vlierberghe, S., Dubruel, P. \& Schacht, E. (2011). Biomacromolecules, 12, 1387-1408.

Vijay, R. J., Melikechi, N., Thomas, T., Gunaseelan, R., Arockiaraj, M. A. \& Sagayaraj, P. (2012). J. Cryst. Growth, 338, 170-176.

Vorotnikov, Y. A., Mikhailov, M. A., Brylev, K. A., Piryazev, D. A., Kuratieva, N. V., Sokolov, M. N., Mironov, Y. V. \& Shestopalov, M. A. (2015). Izv. Akad. Nauk SSSR, Ser. Khim. (Russ. Chem. Bull.), 64, 2591-2596.

Westrip, S. P. (2010). J. Appl. Cryst. 43, 920-925.

Woeste, G., Meyer, W. H. \& Wegner, G. (1993). Makromol. Chem. 194, 1237-1248. 


\section{supporting information}

Acta Cryst. (2019). E75, 946-950 [https://doi.org/10.1107/S2056989019007758]

Structure and Hirshfeld surface analysis of the salt $\mathrm{N}, \mathrm{N}, \mathrm{N}$-trimethyl-1-(4-vinylphenyl)methanaminium 4-vinylbenzenesulfonate

\section{John McAdam, Lyall R. Hanton, Stephen C. Moratti, Jim Simpson and Ravindra N.}

\section{Wickramasinhage}

\section{Computing details}

Data collection: CrysAlis PRO (Rigaku OD, 2018); cell refinement: CrysAlis PRO (Rigaku OD, 2018); data reduction: CrysAlis PRO (Rigaku OD, 2018); program(s) used to solve structure: SHELXT (Sheldrick, 2015a); program(s) used to refine structure: SHELXL2018 (Sheldrick, 2015b) and TITAN (Hunter \& Simpson, 1999); molecular graphics: Mercury (Macrae et al., 2008); software used to prepare material for publication: SHELXL2014 (Sheldrick, 2015b), enCIFer (Allen et al., 2004), PLATON (Spek, 2009), publCIF (Westrip, 2010) and WinGX (Farrugia, 2012).

$\mathrm{N}, \mathrm{N}, \mathrm{N}$-Trimethyl-1-(4-vinylphenyl)methanaminium 4-vinylbenzenesulfonate

Crystal data

$\mathrm{C}_{12} \mathrm{H}_{18} \mathrm{~N}^{+} \cdot \mathrm{C}_{8} \mathrm{H}_{7} \mathrm{O}_{3} \mathrm{~S}^{-}$

$M_{r}=359.47$

Orthorhombic, $P 2{ }_{1} 2_{1} 2_{1}$

$a=8.3344(3) \AA$

$b=10.5937$ (4) $\AA$

$c=21.1228(8) \AA$

$V=1864.98(12) \AA^{3}$

$Z=4$

$F(000)=768$

\section{Data collection}

Rigaku Oxford Diffraction SuperNova, Dual, $\mathrm{Cu}$ at home/near, Atlas diffractometer

Radiation source: micro-focus sealed X-ray tube Detector resolution: 5.1725 pixels $\mathrm{mm}^{-1}$ $\omega$ scans

Absorption correction: multi-scan (CrysAlis PRO; Rigaku OD, 2018)

$T_{\min }=0.911, T_{\max }=1.000$

\section{Refinement}

Refinement on $F^{2}$

Least-squares matrix: full

$R\left[F^{2}>2 \sigma\left(F^{2}\right)\right]=0.040$

$w R\left(F^{2}\right)=0.103$

$S=1.04$

3103 reflections
$D_{\mathrm{x}}=1.280 \mathrm{Mg} \mathrm{m}^{-3}$

$\mathrm{Cu} K \alpha$ radiation, $\lambda=1.54184 \AA$

Cell parameters from 2591 reflections

$\theta=4.2-72.3^{\circ}$

$\mu=1.69 \mathrm{~mm}^{-1}$

$T=100 \mathrm{~K}$

Irregular block, colourless

$0.20 \times 0.18 \times 0.08 \mathrm{~mm}$

4767 measured reflections

3103 independent reflections

2784 reflections with $I>2 \sigma(I)$

$R_{\text {int }}=0.029$

$\theta_{\max }=72.8^{\circ}, \theta_{\min }=4.2^{\circ}$

$h=-6 \rightarrow 10$

$k=-12 \rightarrow 12$

$l=-25 \rightarrow 24$

\section{8 parameters}

10 restraints

Hydrogen site location: inferred from neighbouring sites

$\mathrm{H}$-atom parameters constrained 
$w=1 /\left[\sigma^{2}\left(F_{\mathrm{o}}^{2}\right)+(0.0465 P)^{2}+0.5842 P\right]$

where $P=\left(F_{\mathrm{o}}^{2}+2 F_{\mathrm{c}}^{2}\right) / 3$

$(\Delta / \sigma)_{\max }<0.001$

$\Delta \rho_{\max }=0.37$ e $\AA^{-3}$

$\Delta \rho_{\min }=-0.29$ e $\AA^{-3}$
Absolute structure: Flack $x$ determined using 870 quotients $\left[\left(I^{+}\right)-(I)\right] /\left[\left(I^{+}\right)+(I)\right]$ (Parsons et al., 2013)

Absolute structure parameter: -0.040 (19)

Special details

Geometry. All esds (except the esd in the dihedral angle between two 1.s. planes) are estimated using the full covariance matrix. The cell esds are taken into account individually in the estimation of esds in distances, angles and torsion angles; correlations between esds in cell parameters are only used when they are defined by crystal symmetry. An approximate (isotropic) treatment of cell esds is used for estimating esds involving l.s. planes.

Refinement. The vinyl substituent on the benzene ring of the cation is disordered over two sites with a refined occupancy ratio of 0.542 (11):0.458 (11).

Fractional atomic coordinates and isotropic or equivalent isotropic displacement parameters $\left(\AA^{2}\right)$

\begin{tabular}{|c|c|c|c|c|c|}
\hline & $x$ & $y$ & $z$ & $U_{\text {iso }} * / U_{\text {eq }}$ & Occ. $(<1)$ \\
\hline $\mathrm{O} 1$ & $0.7129(3)$ & $0.3773(2)$ & $0.23060(11)$ & $0.0340(6)$ & \\
\hline $\mathrm{O} 2$ & $0.9047(3)$ & $0.2234(2)$ & $0.19192(11)$ & $0.0324(6)$ & \\
\hline $\mathrm{O} 3$ & $0.6241(3)$ & $0.1666(2)$ & $0.20070(11)$ & $0.0316(6)$ & \\
\hline S1 & $0.73918(10)$ & $0.26835(7)$ & $0.19052(3)$ & $0.0240(2)$ & \\
\hline $\mathrm{C} 1$ & $0.7098(4)$ & $0.3210(3)$ & $0.11156(14)$ & $0.0213(7)$ & \\
\hline $\mathrm{C} 2$ & $0.8167(4)$ & $0.4059(3)$ & $0.08477(16)$ & $0.0254(7)$ & \\
\hline $\mathrm{H} 2$ & 0.901987 & 0.439140 & 0.109530 & $0.030^{*}$ & \\
\hline $\mathrm{C} 3$ & $0.8000(4)$ & $0.4426(3)$ & $0.02186(16)$ & $0.0267(8)$ & \\
\hline $\mathrm{H} 3$ & 0.873464 & 0.501524 & 0.004257 & $0.032 *$ & \\
\hline $\mathrm{C} 4$ & $0.6771(4)$ & $0.3943(3)$ & $-0.01563(16)$ & $0.0259(7)$ & \\
\hline $\mathrm{C} 41$ & $0.6602(5)$ & $0.4228(3)$ & $-0.08413(17)$ & $0.0304(8)$ & \\
\hline $\mathrm{H} 41$ & 0.567919 & 0.390300 & -0.104880 & $0.036^{*}$ & \\
\hline $\mathrm{C} 42$ & $0.7607(5)$ & $0.4885(3)$ & $-0.11868(16)$ & $0.0351(8)$ & \\
\hline $\mathrm{H} 42 \mathrm{~A}$ & 0.854786 & 0.522910 & -0.100025 & $0.042 *$ & \\
\hline H42B & 0.739494 & 0.501740 & -0.162365 & $0.042 *$ & \\
\hline $\mathrm{C} 5$ & $0.5676(4)$ & $0.3129(3)$ & $0.01217(17)$ & $0.0302(8)$ & \\
\hline H5 & 0.480488 & 0.281673 & -0.012223 & $0.036^{*}$ & \\
\hline C6 & $0.5829(4)$ & $0.2760(3)$ & $0.07539(16)$ & $0.0283(8)$ & \\
\hline H6 & 0.506511 & 0.220260 & 0.093620 & $0.034 *$ & \\
\hline $\mathrm{C} 7$ & $0.3486(4)$ & $0.3494(4)$ & $0.39999(17)$ & $0.0282(8)$ & \\
\hline $\mathrm{C} 8$ & $0.2924(5)$ & $0.4592(4)$ & $0.4279(2)$ & $0.0391(10)$ & \\
\hline $\mathrm{H} 8$ & 0.270082 & 0.530860 & 0.402275 & $0.047^{*}$ & \\
\hline C9 & $0.2687(6)$ & $0.4656(5)$ & $0.4924(2)$ & 0.0577 (14) & \\
\hline H9 & 0.228496 & 0.541594 & 0.510296 & $0.069^{*}$ & \\
\hline $\mathrm{C} 10$ & $0.3017(5)$ & $0.3649(6)$ & $0.5319(2)$ & $0.0600(15)$ & \\
\hline C101 & $0.2819(10)$ & $0.3361(8)$ & $0.6023(3)$ & $0.038(2)$ & $0.542(11)$ \\
\hline H101 & 0.324589 & 0.260969 & 0.620146 & $0.046^{*}$ & $0.542(11)$ \\
\hline C102 & $0.2045(14)$ & $0.4175(8)$ & $0.6374(4)$ & $0.066(4)$ & $0.542(11)$ \\
\hline $\mathrm{H} 10 \mathrm{~A}$ & 0.162395 & 0.492277 & 0.618983 & $0.080^{*}$ & $0.542(11)$ \\
\hline H10B & 0.190496 & 0.401869 & 0.681348 & $0.080^{*}$ & $0.542(11)$ \\
\hline C103 & $0.2724(12)$ & $0.4160(9)$ & $0.5973(3)$ & $0.033(2)$ & $0.458(11)$ \\
\hline H103 & 0.251488 & 0.503001 & 0.604321 & $0.040^{*}$ & $0.458(11)$ \\
\hline
\end{tabular}




\begin{tabular}{|c|c|c|c|c|c|}
\hline $\mathrm{C} 104$ & $0.2771(11)$ & $0.3345(9)$ & $0.6444(4)$ & $0.038(3)$ & $0.458(11)$ \\
\hline $\mathrm{H} 10 \mathrm{C}$ & 0.298320 & 0.247947 & 0.636175 & $0.045^{*}$ & $0.458(11)$ \\
\hline H10D & 0.259356 & 0.362379 & 0.686556 & $0.045^{*}$ & $0.458(11)$ \\
\hline C11 & $0.3597(5)$ & $0.2564(6)$ & $0.5043(2)$ & $0.0538(13)$ & \\
\hline H11 & 0.384283 & 0.185948 & 0.530359 & $0.065 *$ & \\
\hline $\mathrm{C} 12$ & $0.3830(5)$ & $0.2473(4)$ & 0.43947 (19) & $0.0385(9)$ & \\
\hline H12 & 0.422829 & 0.171040 & 0.421795 & $0.046^{*}$ & \\
\hline $\mathrm{C} 13$ & $0.3803(4)$ & $0.3451(4)$ & $0.32982(17)$ & $0.0329(8)$ & \\
\hline H13A & 0.468168 & 0.284452 & 0.321549 & $0.039 *$ & \\
\hline H13B & 0.417035 & 0.429445 & 0.315753 & $0.039 *$ & \\
\hline $\mathrm{C} 14$ & $0.2826(5)$ & $0.3111(5)$ & $0.22225(17)$ & $0.0528(13)$ & \\
\hline H14A & 0.190237 & 0.290203 & 0.195519 & $0.079 *$ & \\
\hline H14B & 0.368715 & 0.249929 & 0.214829 & $0.079 *$ & \\
\hline $\mathrm{H} 14 \mathrm{C}$ & 0.320828 & 0.396094 & 0.211787 & $0.079 *$ & \\
\hline $\mathrm{C} 15$ & $0.0986(4)$ & $0.3955(3)$ & 0.30061 (19) & $0.0330(8)$ & \\
\hline $\mathrm{H} 15 \mathrm{~A}$ & 0.010714 & 0.374402 & 0.271685 & $0.050^{*}$ & \\
\hline $\mathrm{H} 15 \mathrm{~B}$ & 0.134872 & 0.481981 & 0.292401 & $0.050^{*}$ & \\
\hline $\mathrm{H} 15 \mathrm{C}$ & 0.061038 & 0.388818 & 0.344442 & $0.050^{*}$ & \\
\hline N1 & 0.2336 & $0.3069(3)$ & $0.29050(13)$ & $0.0289(7)$ & \\
\hline C16 & $0.1808(5)$ & $0.1749(3)$ & $0.3060(2)$ & $0.0444(10)$ & \\
\hline H16A & 0.148219 & 0.170607 & 0.350491 & $0.067^{*}$ & \\
\hline H16B & 0.269731 & 0.116280 & 0.298539 & $0.067^{*}$ & \\
\hline $\mathrm{H} 16 \mathrm{C}$ & 0.089814 & 0.151749 & 0.278940 & $0.067 *$ & \\
\hline
\end{tabular}

Atomic displacement parameters $\left(\AA^{2}\right)$

\begin{tabular}{|c|c|c|c|c|c|c|}
\hline & $U^{11}$ & $U^{22}$ & $U^{33}$ & $U^{12}$ & $U^{13}$ & $U^{23}$ \\
\hline $\mathrm{O} 1$ & $0.0421(16)$ & $0.0313(13)$ & $0.0287(12)$ & $0.0004(12)$ & $0.0005(11)$ & $-0.0081(10)$ \\
\hline $\mathrm{O} 2$ & $0.0250(12)$ & $0.0454(14)$ & $0.0269(12)$ & $0.0102(11)$ & $-0.0034(10)$ & $-0.0010(13)$ \\
\hline $\mathrm{O} 3$ & $0.0370(14)$ & $0.0303(13)$ & $0.0275(13)$ & $-0.0076(12)$ & $-0.0008(11)$ & $0.0046(11)$ \\
\hline $\mathrm{S} 1$ & $0.0258(4)$ & $0.0259(4)$ & $0.0203(3)$ & $0.0004(4)$ & -0.0009 & $-0.0022(3)$ \\
\hline $\mathrm{C} 1$ & $0.0243(17)$ & $0.0198(14)$ & $0.0198(14)$ & $0.0036(14)$ & $-0.0016(13)$ & $-0.0010(12)$ \\
\hline $\mathrm{C} 2$ & $0.0218(16)$ & $0.0237(16)$ & 0.0307 (17) & $-0.0006(15)$ & $-0.0001(14)$ & $-0.0042(14)$ \\
\hline $\mathrm{C} 3$ & $0.0259(18)$ & $0.0232(16)$ & $0.0311(17)$ & $-0.0003(15)$ & $0.0064(15)$ & $0.0011(14)$ \\
\hline $\mathrm{C} 4$ & 0.0283 (18) & $0.0244(16)$ & $0.0249(17)$ & $0.0043(15)$ & $-0.0011(14)$ & $-0.0019(14)$ \\
\hline C41 & $0.035(2)$ & $0.0286(18)$ & $0.0280(18)$ & 0.0035 (17) & $-0.0043(16)$ & $0.0003(15)$ \\
\hline $\mathrm{C} 42$ & $0.035(2)$ & 0.0423 (19) & $0.0275(17)$ & $0.005(2)$ & $0.0014(18)$ & $0.0049(15)$ \\
\hline C5 & $0.0280(18)$ & 0.0302 (18) & 0.0324 (19) & $-0.0023(16)$ & $-0.0100(15)$ & $0.0016(15)$ \\
\hline C6 & $0.0283(18)$ & $0.0257(17)$ & 0.0308 (18) & $-0.0034(16)$ & $-0.0057(14)$ & $0.0030(16)$ \\
\hline $\mathrm{C} 7$ & $0.0211(17)$ & 0.0344 (19) & $0.0292(18)$ & $-0.0060(16)$ & $-0.0032(14)$ & $-0.0010(16)$ \\
\hline $\mathrm{C} 8$ & $0.034(2)$ & $0.038(2)$ & $0.046(2)$ & $0.0006(18)$ & $-0.0138(18)$ & -0.0090 \\
\hline C9 & $0.034(2)$ & $0.088(4)$ & $0.051(3)$ & $0.008(3)$ & $-0.013(2)$ & $-0.036(3)$ \\
\hline $\mathrm{C} 10$ & $0.031(2)$ & $0.116(5)$ & $0.034(2)$ & -0.018 & $-0.0053(18)$ & $-0.012(3)$ \\
\hline C101 & $0.045(5)$ & $0.032(5)$ & $0.038(5)$ & $-0.016(4)$ & $-0.016(4)$ & 0.009 (4) \\
\hline C102 & $0.126(11)$ & $0.043(5)$ & $0.030(5)$ & -0.009 (6) & $-0.009(6)$ & $0.005(4)$ \\
\hline C103 & $0.040(5)$ & $0.031(5)$ & $0.028(5)$ & $-0.003(5)$ & $-0.002(4)$ & $-0.001(4)$ \\
\hline C104 & $0.044(6)$ & $0.045(6)$ & $0.023(5)$ & $-0.010(5)$ & $0.002(4)$ & $0.002(4)$ \\
\hline $\mathrm{C} 11$ & $0.048(3)$ & $0.073(3)$ & $0.040(2)$ & $-0.026(3)$ & -0.0168 (19) & $0.025(2)$ \\
\hline
\end{tabular}




$\begin{array}{lllllll}\mathrm{C} 12 & 0.036(2) & 0.033(2) & 0.046(2) & -0.007(2) & -0.0098(17) & 0.0038(18) \\ \mathrm{C} 13 & 0.0223(17) & 0.042(2) & 0.0347(19) & -0.0019(17) & -0.0027(15) & 0.0019(17) \\ \mathrm{C} 14 & 0.039(2) & 0.094(4) & 0.0250(19) & 0.000(3) & 0.0010(17) & -0.007(2) \\ \mathrm{C} 15 & 0.0293(18) & 0.0291(18) & 0.041(2) & 0.0064(16) & -0.0063(17) & -0.0055(17) \\ \mathrm{N} 1 & 0.0267(15) & 0.0331(15) & 0.0271(14) & 0.0018(14) & -0.0027(13) & -0.0027(11) \\ \mathrm{C} 16 & 0.056(2) & 0.0216(17) & 0.055(3) & -0.0022(18) & -0.019(2) & -0.0020(19)\end{array}$

Geometric parameters $\left(\AA,{ }^{\circ}\right)$

\begin{tabular}{|c|c|c|c|}
\hline $\mathrm{O} 1-\mathrm{S} 1$ & $1.448(2)$ & $\mathrm{C} 10-\mathrm{C} 101$ & $1.527(7)$ \\
\hline $\mathrm{O} 2-\mathrm{S} 1$ & $1.460(2)$ & $\mathrm{C} 101-\mathrm{C} 102$ & $1.307(9)$ \\
\hline $\mathrm{O} 3-\mathrm{S} 1$ & $1.459(2)$ & C101-H101 & 0.9500 \\
\hline $\mathrm{S} 1-\mathrm{C} 1$ & $1.776(3)$ & $\mathrm{C} 102-\mathrm{H} 10 \mathrm{~A}$ & 0.9500 \\
\hline $\mathrm{C} 1-\mathrm{C} 2$ & $1.387(4)$ & $\mathrm{C} 102-\mathrm{H} 10 \mathrm{~B}$ & 0.9500 \\
\hline $\mathrm{C} 1-\mathrm{C} 6$ & $1.389(5)$ & $\mathrm{C} 103-\mathrm{C} 104$ & $1.317(9)$ \\
\hline $\mathrm{C} 2-\mathrm{C} 3$ & $1.392(5)$ & $\mathrm{C} 103-\mathrm{H} 103$ & 0.9500 \\
\hline $\mathrm{C} 2-\mathrm{H} 2$ & 0.9500 & $\mathrm{C} 104-\mathrm{H} 10 \mathrm{C}$ & 0.9500 \\
\hline $\mathrm{C} 3-\mathrm{C} 4$ & $1.392(5)$ & $\mathrm{C} 104-\mathrm{H} 10 \mathrm{D}$ & 0.9500 \\
\hline $\mathrm{C} 3-\mathrm{H} 3$ & 0.9500 & $\mathrm{C} 11-\mathrm{C} 12$ & $1.387(6)$ \\
\hline $\mathrm{C} 4-\mathrm{C} 5$ & $1.386(5)$ & C11-H11 & 0.9500 \\
\hline $\mathrm{C} 4-\mathrm{C} 41$ & $1.485(5)$ & $\mathrm{C} 12-\mathrm{H} 12$ & 0.9500 \\
\hline $\mathrm{C} 41-\mathrm{C} 42$ & $1.311(5)$ & $\mathrm{C} 13-\mathrm{N} 1$ & $1.533(5)$ \\
\hline $\mathrm{C} 41-\mathrm{H} 41$ & 0.9500 & $\mathrm{C} 13-\mathrm{H} 13 \mathrm{~A}$ & 0.9900 \\
\hline $\mathrm{C} 42-\mathrm{H} 42 \mathrm{~A}$ & 0.9500 & $\mathrm{C} 13-\mathrm{H} 13 \mathrm{~B}$ & 0.9900 \\
\hline $\mathrm{C} 42-\mathrm{H} 42 \mathrm{~B}$ & 0.9500 & $\mathrm{C} 14-\mathrm{N} 1$ & 1.499 (4) \\
\hline $\mathrm{C} 5-\mathrm{C} 6$ & $1.397(4)$ & $\mathrm{C} 14-\mathrm{H} 14 \mathrm{~A}$ & 0.9800 \\
\hline $\mathrm{C} 5-\mathrm{H} 5$ & 0.9500 & C14-H14B & 0.9800 \\
\hline C6- $-\mathrm{H} 6$ & 0.9500 & $\mathrm{C} 14-\mathrm{H} 14 \mathrm{C}$ & 0.9800 \\
\hline $\mathrm{C} 7-\mathrm{C} 8$ & $1.386(5)$ & $\mathrm{C} 15-\mathrm{N} 1$ & $1.481(4)$ \\
\hline $\mathrm{C} 7-\mathrm{C} 12$ & $1.395(5)$ & C15-H15A & 0.9800 \\
\hline $\mathrm{C} 7-\mathrm{C} 13$ & $1.506(5)$ & C15-H15B & 0.9800 \\
\hline $\mathrm{C} 8-\mathrm{C} 9$ & $1.380(6)$ & $\mathrm{C} 15-\mathrm{H} 15 \mathrm{C}$ & 0.9800 \\
\hline $\mathrm{C} 8-\mathrm{H} 8$ & 0.9500 & $\mathrm{~N} 1-\mathrm{C} 16$ & $1.501(4)$ \\
\hline $\mathrm{C} 9-\mathrm{C} 10$ & $1.382(8)$ & C16-H16A & 0.9800 \\
\hline C9-H9 & 0.9500 & C16-H16B & 0.9800 \\
\hline $\mathrm{C} 10-\mathrm{C} 11$ & $1.376(8)$ & $\mathrm{C} 16-\mathrm{H} 16 \mathrm{C}$ & 0.9800 \\
\hline $\mathrm{C} 10-\mathrm{C} 103$ & $1.504(7)$ & & \\
\hline $\mathrm{O} 1-\mathrm{S} 1-\mathrm{O} 3$ & $113.77(15)$ & $\mathrm{C} 10-\mathrm{C} 101-\mathrm{H} 101$ & 120.8 \\
\hline $\mathrm{O} 1-\mathrm{S} 1-\mathrm{O} 2$ & $113.04(15)$ & $\mathrm{C} 101-\mathrm{C} 102-\mathrm{H} 10 \mathrm{~A}$ & 120.0 \\
\hline $\mathrm{O} 3-\mathrm{S} 1-\mathrm{O} 2$ & $112.16(16)$ & $\mathrm{C} 101-\mathrm{C} 102-\mathrm{H} 10 \mathrm{~B}$ & 120.0 \\
\hline $\mathrm{O} 1-\mathrm{S} 1-\mathrm{C} 1$ & $106.15(14)$ & $\mathrm{H} 10 \mathrm{~A}-\mathrm{C} 102-\mathrm{H} 10 \mathrm{~B}$ & 120.0 \\
\hline $\mathrm{O} 3-\mathrm{S} 1-\mathrm{C} 1$ & $106.25(15)$ & $\mathrm{C} 104-\mathrm{C} 103-\mathrm{C} 10$ & $116.9(8)$ \\
\hline $\mathrm{O} 2-\mathrm{S} 1-\mathrm{C} 1$ & $104.59(15)$ & $\mathrm{C} 104-\mathrm{C} 103-\mathrm{H} 103$ & 121.5 \\
\hline $\mathrm{C} 2-\mathrm{C} 1-\mathrm{C} 6$ & $119.2(3)$ & $\mathrm{C} 10-\mathrm{C} 103-\mathrm{H} 103$ & 121.5 \\
\hline $\mathrm{C} 2-\mathrm{C} 1-\mathrm{S} 1$ & $119.9(2)$ & $\mathrm{C} 103-\mathrm{C} 104-\mathrm{H} 10 \mathrm{C}$ & 120.0 \\
\hline $\mathrm{C} 6-\mathrm{C} 1-\mathrm{S} 1$ & $120.9(3)$ & $\mathrm{C} 103-\mathrm{C} 104-\mathrm{H} 10 \mathrm{D}$ & 120.0 \\
\hline $\mathrm{C} 1-\mathrm{C} 2-\mathrm{C} 3$ & $120.4(3)$ & $\mathrm{H} 10 \mathrm{C}-\mathrm{C} 104-\mathrm{H} 10 \mathrm{D}$ & 120.0 \\
\hline
\end{tabular}




\begin{tabular}{|c|c|c|c|}
\hline $\mathrm{C} 1-\mathrm{C} 2-\mathrm{H} 2$ & 119.8 & $\mathrm{C} 10-\mathrm{C} 11-\mathrm{C} 12$ & $121.7(5)$ \\
\hline $\mathrm{C} 3-\mathrm{C} 2-\mathrm{H} 2$ & 119.8 & $\mathrm{C} 10-\mathrm{C} 11-\mathrm{H} 11$ & 119.1 \\
\hline $\mathrm{C} 2-\mathrm{C} 3-\mathrm{C} 4$ & $120.9(3)$ & $\mathrm{C} 12-\mathrm{C} 11-\mathrm{H} 11$ & 119.1 \\
\hline $\mathrm{C} 2-\mathrm{C} 3-\mathrm{H} 3$ & 119.5 & $\mathrm{C} 11-\mathrm{C} 12-\mathrm{C} 7$ & $120.5(4)$ \\
\hline $\mathrm{C} 4-\mathrm{C} 3-\mathrm{H} 3$ & 119.5 & $\mathrm{C} 11-\mathrm{C} 12-\mathrm{H} 12$ & 119.8 \\
\hline $\mathrm{C} 5-\mathrm{C} 4-\mathrm{C} 3$ & $118.2(3)$ & $\mathrm{C} 7-\mathrm{C} 12-\mathrm{H} 12$ & 119.8 \\
\hline $\mathrm{C} 5-\mathrm{C} 4-\mathrm{C} 41$ & $118.5(3)$ & $\mathrm{C} 7-\mathrm{C} 13-\mathrm{N} 1$ & $113.7(3)$ \\
\hline $\mathrm{C} 3-\mathrm{C} 4-\mathrm{C} 41$ & $123.3(3)$ & $\mathrm{C} 7-\mathrm{C} 13-\mathrm{H} 13 \mathrm{~A}$ & 108.8 \\
\hline $\mathrm{C} 42-\mathrm{C} 41-\mathrm{C} 4$ & $126.1(4)$ & $\mathrm{N} 1-\mathrm{C} 13-\mathrm{H} 13 \mathrm{~A}$ & 108.8 \\
\hline $\mathrm{C} 42-\mathrm{C} 41-\mathrm{H} 41$ & 116.9 & $\mathrm{C} 7-\mathrm{C} 13-\mathrm{H} 13 \mathrm{~B}$ & 108.8 \\
\hline $\mathrm{C} 4-\mathrm{C} 41-\mathrm{H} 41$ & 116.9 & $\mathrm{~N} 1-\mathrm{C} 13-\mathrm{H} 13 \mathrm{~B}$ & 108.8 \\
\hline $\mathrm{C} 41-\mathrm{C} 42-\mathrm{H} 42 \mathrm{~A}$ & 120.0 & $\mathrm{H} 13 \mathrm{~A}-\mathrm{C} 13-\mathrm{H} 13 \mathrm{~B}$ & 107.7 \\
\hline $\mathrm{C} 41-\mathrm{C} 42-\mathrm{H} 42 \mathrm{~B}$ & 120.0 & $\mathrm{~N} 1-\mathrm{C} 14-\mathrm{H} 14 \mathrm{~A}$ & 109.5 \\
\hline $\mathrm{H} 42 \mathrm{~A}-\mathrm{C} 42-\mathrm{H} 42 \mathrm{~B}$ & 120.0 & $\mathrm{~N} 1-\mathrm{C} 14-\mathrm{H} 14 \mathrm{~B}$ & 109.5 \\
\hline $\mathrm{C} 4-\mathrm{C} 5-\mathrm{C} 6$ & $121.3(3)$ & $\mathrm{H} 14 \mathrm{~A}-\mathrm{C} 14-\mathrm{H} 14 \mathrm{~B}$ & 109.5 \\
\hline $\mathrm{C} 4-\mathrm{C} 5-\mathrm{H} 5$ & 119.4 & $\mathrm{~N} 1-\mathrm{C} 14-\mathrm{H} 14 \mathrm{C}$ & 109.5 \\
\hline $\mathrm{C} 6-\mathrm{C} 5-\mathrm{H} 5$ & 119.4 & $\mathrm{H} 14 \mathrm{~A}-\mathrm{C} 14-\mathrm{H} 14 \mathrm{C}$ & 109.5 \\
\hline $\mathrm{C} 1-\mathrm{C} 6-\mathrm{C} 5$ & $119.9(3)$ & $\mathrm{H} 14 \mathrm{~B}-\mathrm{C} 14-\mathrm{H} 14 \mathrm{C}$ & 109.5 \\
\hline $\mathrm{C} 1-\mathrm{C} 6-\mathrm{H} 6$ & 120.0 & $\mathrm{~N} 1-\mathrm{C} 15-\mathrm{H} 15 \mathrm{~A}$ & 109.5 \\
\hline $\mathrm{C} 5-\mathrm{C} 6-\mathrm{H} 6$ & 120.0 & $\mathrm{~N} 1-\mathrm{C} 15-\mathrm{H} 15 \mathrm{~B}$ & 109.5 \\
\hline $\mathrm{C} 8-\mathrm{C} 7-\mathrm{C} 12$ & $117.8(4)$ & $\mathrm{H} 15 \mathrm{~A}-\mathrm{C} 15-\mathrm{H} 15 \mathrm{~B}$ & 109.5 \\
\hline $\mathrm{C} 8-\mathrm{C} 7-\mathrm{C} 13$ & $120.1(4)$ & $\mathrm{N} 1-\mathrm{C} 15-\mathrm{H} 15 \mathrm{C}$ & 109.5 \\
\hline $\mathrm{C} 12-\mathrm{C} 7-\mathrm{C} 13$ & $121.9(4)$ & $\mathrm{H} 15 \mathrm{~A}-\mathrm{C} 15-\mathrm{H} 15 \mathrm{C}$ & 109.5 \\
\hline $\mathrm{C} 9-\mathrm{C} 8-\mathrm{C} 7$ & $120.6(4)$ & $\mathrm{H} 15 \mathrm{~B}-\mathrm{C} 15-\mathrm{H} 15 \mathrm{C}$ & 109.5 \\
\hline $\mathrm{C} 9-\mathrm{C} 8-\mathrm{H} 8$ & 119.7 & $\mathrm{C} 15-\mathrm{N} 1-\mathrm{C} 16$ & $109.7(3)$ \\
\hline $\mathrm{C} 7-\mathrm{C} 8-\mathrm{H} 8$ & 119.7 & $\mathrm{C} 15-\mathrm{N} 1-\mathrm{C} 14$ & $109.1(3)$ \\
\hline $\mathrm{C} 8-\mathrm{C} 9-\mathrm{C} 10$ & $122.0(5)$ & $\mathrm{C} 16-\mathrm{N} 1-\mathrm{C} 14$ & $108.5(3)$ \\
\hline $\mathrm{C} 8-\mathrm{C} 9-\mathrm{H} 9$ & 119.0 & $\mathrm{C} 15-\mathrm{N} 1-\mathrm{C} 13$ & $111.1(3)$ \\
\hline $\mathrm{C} 10-\mathrm{C} 9-\mathrm{H} 9$ & 119.0 & $\mathrm{C} 16-\mathrm{N} 1-\mathrm{C} 13$ & $111.2(3)$ \\
\hline $\mathrm{C} 11-\mathrm{C} 10-\mathrm{C} 9$ & $117.4(4)$ & $\mathrm{C} 14-\mathrm{N} 1-\mathrm{C} 13$ & $107.2(3)$ \\
\hline $\mathrm{C} 11-\mathrm{C} 10-\mathrm{C} 103$ & $138.3(6)$ & $\mathrm{N} 1-\mathrm{C} 16-\mathrm{H} 16 \mathrm{~A}$ & 109.5 \\
\hline $\mathrm{C} 9-\mathrm{C} 10-\mathrm{C} 103$ & $104.1(6)$ & $\mathrm{N} 1-\mathrm{C} 16-\mathrm{H} 16 \mathrm{~B}$ & 109.5 \\
\hline $\mathrm{C} 11-\mathrm{C} 10-\mathrm{C} 101$ & $106.5(6)$ & $\mathrm{H} 16 \mathrm{~A}-\mathrm{C} 16-\mathrm{H} 16 \mathrm{~B}$ & 109.5 \\
\hline $\mathrm{C} 9-\mathrm{C} 10-\mathrm{C} 101$ & $136.0(6)$ & $\mathrm{N} 1-\mathrm{C} 16-\mathrm{H} 16 \mathrm{C}$ & 109.5 \\
\hline $\mathrm{C} 102-\mathrm{C} 101-\mathrm{C} 10$ & $118.3(8)$ & $\mathrm{H} 16 \mathrm{~A}-\mathrm{C} 16-\mathrm{H} 16 \mathrm{C}$ & 109.5 \\
\hline $\mathrm{C} 102-\mathrm{C} 101-\mathrm{H} 101$ & 120.8 & $\mathrm{H} 16 \mathrm{~B}-\mathrm{C} 16-\mathrm{H} 16 \mathrm{C}$ & 109.5 \\
\hline $\mathrm{O} 1-\mathrm{S} 1-\mathrm{C} 1-\mathrm{C} 2$ & $67.5(3)$ & $\mathrm{C} 7-\mathrm{C} 8-\mathrm{C} 9-\mathrm{C} 10$ & $1.0(7)$ \\
\hline $\mathrm{O} 3-\mathrm{S} 1-\mathrm{C} 1-\mathrm{C} 2$ & $-171.1(2)$ & $\mathrm{C} 8-\mathrm{C} 9-\mathrm{C} 10-\mathrm{C} 11$ & $-0.1(7)$ \\
\hline $\mathrm{O} 2-\mathrm{S} 1-\mathrm{C} 1-\mathrm{C} 2$ & $-52.3(3)$ & $\mathrm{C} 8-\mathrm{C} 9-\mathrm{C} 10-\mathrm{C} 103$ & $175.4(6)$ \\
\hline $\mathrm{O} 1-\mathrm{S} 1-\mathrm{C} 1-\mathrm{C} 6$ & $-114.1(3)$ & $\mathrm{C} 8-\mathrm{C} 9-\mathrm{C} 10-\mathrm{C} 101$ & $-175.7(6)$ \\
\hline $\mathrm{O} 3-\mathrm{S} 1-\mathrm{C} 1-\mathrm{C} 6$ & $7.3(3)$ & $\mathrm{C} 11-\mathrm{C} 10-\mathrm{C} 101-\mathrm{C} 102$ & $-169.3(7)$ \\
\hline $\mathrm{O} 2-\mathrm{S} 1-\mathrm{C} 1-\mathrm{C} 6$ & $126.1(3)$ & $\mathrm{C} 9-\mathrm{C} 10-\mathrm{C} 101-\mathrm{C} 102$ & $6.6(12)$ \\
\hline $\mathrm{C} 6-\mathrm{C} 1-\mathrm{C} 2-\mathrm{C} 3$ & $-1.9(5)$ & $\mathrm{C} 11-\mathrm{C} 10-\mathrm{C} 103-\mathrm{C} 104$ & $-13.7(13)$ \\
\hline $\mathrm{S} 1-\mathrm{C} 1-\mathrm{C} 2-\mathrm{C} 3$ & $176.5(3)$ & $\mathrm{C} 9-\mathrm{C} 10-\mathrm{C} 103-\mathrm{C} 104$ & $172.3(8)$ \\
\hline $\mathrm{C} 1-\mathrm{C} 2-\mathrm{C} 3-\mathrm{C} 4$ & $-0.7(5)$ & $\mathrm{C} 9-\mathrm{C} 10-\mathrm{C} 11-\mathrm{C} 12$ & $-0.6(7)$ \\
\hline $\mathrm{C} 2-\mathrm{C} 3-\mathrm{C} 4-\mathrm{C} 5$ & $2.9(5)$ & $\mathrm{C} 103-\mathrm{C} 10-\mathrm{C} 11-\mathrm{C} 12$ & $-174.0(7)$ \\
\hline $\mathrm{C} 2-\mathrm{C} 3-\mathrm{C} 4-\mathrm{C} 41$ & $-175.4(3)$ & $\mathrm{C} 101-\mathrm{C} 10-\mathrm{C} 11-\mathrm{C} 12$ & $176.3(5)$ \\
\hline
\end{tabular}




$\begin{array}{llll}\mathrm{C} 5-\mathrm{C} 4-\mathrm{C} 41-\mathrm{C} 42 & -173.8(4) & \mathrm{C} 10-\mathrm{C} 11-\mathrm{C} 12-\mathrm{C} 7 & 0.2(6) \\ \mathrm{C} 3-\mathrm{C} 4-\mathrm{C} 41-\mathrm{C} 42 & 4.5(6) & \mathrm{C} 8-\mathrm{C} 7-\mathrm{C} 12-\mathrm{C} 11 & 0.7(5) \\ \mathrm{C} 3-\mathrm{C} 4-\mathrm{C} 5-\mathrm{C} 6 & -2.5(5) & \mathrm{C} 13-\mathrm{C} 7-\mathrm{C} 12-\mathrm{C} 11 & 176.9(4) \\ \mathrm{C} 41-\mathrm{C} 4-\mathrm{C} 5-\mathrm{C} 6 & 175.9(3) & \mathrm{C} 8-\mathrm{C} 7-\mathrm{C} 13-\mathrm{N} 1 & -88.7(4) \\ \mathrm{C} 2-\mathrm{C} 1-\mathrm{C} 6-\mathrm{C} 5 & 2.3(5) & \mathrm{C} 12-\mathrm{C} 7-\mathrm{C} 13-\mathrm{N} 1 & 95.2(4) \\ \mathrm{S} 1-\mathrm{C} 1-\mathrm{C} 6-\mathrm{C} 5 & -176.2(3) & \mathrm{C} 7-\mathrm{C} 13-\mathrm{N} 1-\mathrm{C} 15 & 59.1(4) \\ \mathrm{C} 4-\mathrm{C} 5-\mathrm{C} 6-\mathrm{C} 1 & -0.1(6) & \mathrm{C} 7-\mathrm{C} 13-\mathrm{N} 1-\mathrm{C} 16 & -63.3(4) \\ \mathrm{C} 12-\mathrm{C} 7-\mathrm{C} 8-\mathrm{C} 9 & -1.3(6) & \mathrm{C} 7-\mathrm{C} 13-\mathrm{N} 1-\mathrm{C} 14 & 178.2(3) \\ \mathrm{C} 13-\mathrm{C} 7-\mathrm{C} 8-\mathrm{C} 9 & -177.6(4) & & \end{array}$

Hydrogen-bond geometry $\left(A,{ }^{\circ}\right)$

$C g 1$ is the centroid of the $\mathrm{C} 1-\mathrm{C} 6$ benzene ring.

\begin{tabular}{lllll}
\hline$D-\mathrm{H} \cdots A$ & $D-\mathrm{H}$ & $\mathrm{H} \cdots A$ & $D \cdots A$ & $D-\mathrm{H} \cdots A$ \\
\hline $\mathrm{C} 14-\mathrm{H} 14 B \cdots \mathrm{O} 3$ & 0.98 & 2.32 & $3.264(5)$ & 161 \\
$\mathrm{C} 14-\mathrm{H} 14 A \cdots \mathrm{O} 2^{\mathrm{i}}$ & 0.98 & 2.48 & $3.346(5)$ & 147 \\
$\mathrm{C} 15-\mathrm{H} 15 A \cdots \mathrm{O} 1^{\mathrm{i}}$ & 0.98 & 2.63 & $3.544(4)$ & 155 \\
$\mathrm{C} 15-\mathrm{H} 15 A \cdots \mathrm{O} 2^{\mathrm{i}}$ & 0.98 & 2.49 & $3.348(4)$ & 147 \\
$\mathrm{C} 13-\mathrm{H} 13 B \cdots \mathrm{O} 3^{\mathrm{ii}}$ & 0.99 & 2.56 & $3.466(5)$ & 152 \\
$\mathrm{C} 15-\mathrm{H} 15 B \cdots \mathrm{O} 2^{\mathrm{ii}}$ & 0.98 & 2.60 & $3.477(4)$ & 149 \\
$\mathrm{C} 16-\mathrm{H} 16 B \cdots \mathrm{O} 1^{\mathrm{iii}}$ & 0.98 & 2.61 & $3.365(4)$ & 134 \\
$\mathrm{C} 16-\mathrm{H} 16 C \cdots \mathrm{O} 2^{\mathrm{i}}$ & 0.98 & 2.52 & $3.370(5)$ & 146 \\
$\mathrm{C} 41-\mathrm{H} 41 \cdots \mathrm{O} 2^{\mathrm{iv}}$ & 0.95 & 2.58 & $3.481(4)$ & 157 \\
$\mathrm{C} 42-\mathrm{H} 42 B \cdots \mathrm{O} 1^{\mathrm{v}}$ & 0.95 & 2.63 & $3.494(4)$ & 151 \\
$\mathrm{C} 5-\mathrm{H} 5 \cdots C g 1^{\mathrm{iv}}$ & 0.95 & 2.93 & $3.837(4)$ & 161 \\
& & & &
\end{tabular}

Symmetry codes: (i) $x-1, y, z$; (ii) $-x+1, y+1 / 2,-z+1 / 2$; (iii) $-x+1, y-1 / 2,-z+1 / 2$; (iv) $x-1 / 2,-y+1 / 2,-z$; (v) $-x+3 / 2,-y+1, z-1 / 2$. 